\title{
Antecedents of patient health outcomes in dialysis clinics: a national study
}

\author{
Chenzhang Bao and Indranil Bardhan \\ Jindal School of Management, The University of Texas at Dallas, \\ Richardson, Texas, USA
}

\begin{abstract}
Purpose - The purpose of this study is to evaluate the determinants of health outcomes of dialysis patients, while specifically focusing on the role of dialysis process measures and dialysis practice characteristics. The dialysis industry is facing a major transition from a volume-based health care system to a value-based cost-efficient care model, in the USA. Under the bundled Prospective Payment System, the treatment-based payment model is subject to meeting quality thresholds as defined by clinical process measures including dialysis adequacy and anemia management. Few studies have focused on studying these two processes and their association with the quality of patient health outcomes.

Design/methodology/approach - In this study, the authors focus on identifying the determinants of patient health outcomes among freestanding dialysis clinics, using a large cross-sectional data set of 4,571 dialysis clinics in the USA. The authors use econometric analyses to estimate the association between dialysis facility characteristics and practice patterns and their association with dialysis process measures and hospitalization risk. Findings - The authors find that reusing dialyzers and increasing the number of dialysis stations is associated with higher levels of clinical quality. This research indicates that deploying more nurses on-site allows patients to avail adequate dialysis, while increasing the supply of physicians can hurt anemia control process. In addition, the authors report that offering peritoneal dialysis and late night shifts are not beneficial practices in terms of their impact on the hospitalization risk.
\end{abstract}

Research limitations/implications - While early studies of dialysis care mainly focused on the associations between practice patterns and patient outcomes, this research reveals the underlying mechanisms of these relationships by exploring the mediation effects of clinical dialysis processes on patient outcomes. The results indicate that dialysis process measures mediate the impact of the operational characteristics of dialysis centers on patient hospitalization rates.

Practical implications - This study offers several managerial insights for owners and operators of dialysis clinics with respect to the association between managerial and clinical practices that they deploy within dialysis clinics and their impact on clinical quality measures as well as hospitalization risk of patients. Managers can draw on this study to optimize staffing levels in their dialysis clinics, and implement innovative clinical practices.

Social implications - Considering the growth in healthcare expenditures in developing and developed countries, and specifically for costly diagnoses such as dialyses, this study offers several insights related to the inter-relationships between dialysis practice patterns and their clinical quality measures.

Originality/value - This study makes several major contributions. First, the authors address the extant gap in the literature on the relationships between dialysis facility and practice characteristics and clinical outcomes, while specifically highlighting the role of clinical process measures as antecedents of patient hospitalization ratio, a key metric used to measure performance of dialysis clinics. Second, this study sheds

(C) Chenzhang Bao and Indranil Bardhan. Published in Journal of Centrum Cathedra: The Business and Economics Research Journal. Published by Emerald Publishing Limited. This article is published under the Creative Commons Attribution (CC BY 4.0) licence. Anyone may reproduce, distribute, translate and create derivative works of this article (for both commercial \& non-commercial purposes), subject to full attribution to the original publication and authors. The full terms of this licence may be seen at http://creativecommons.org/licenses/by/4.0/legalcode

This article is part of a special issue Guest Edited by Rajiv D. Banker and Vincent Charles.

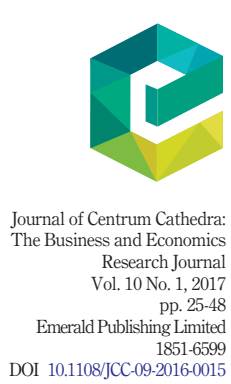


JCC

10,1

26

light on the underlying mechanisms that serve as enablers of the dialysis adequacy and anemia management. To the best of the authors' knowledge, this is the first study to explore these relationships in the dialysis industry. The authors' approach provides a new direction for future studies to explore the pathways that may impact clinical quality measures in the delivery of dialysis services.

Keywords Econometrics, Clinical quality, Dialysis, Hospitalization, Practice patterns

Paper type Research paper

\section{Introduction}

During the past two decades, the number of end-stage renal disease (ESRD) cases has continued to rise, and the number of patients needing hemodialysis (HD) and peritoneal dialysis (PD) has risen to 600,000, according to the USA Renal Data System Annual Data Report[1]. In 2013, ESRD beneficiaries comprised 1.2 per cent of the Medicare population and accounted for an estimated 6.3 per cent of total Medicare spending, totaling over $\$ 30.9 \mathrm{bn}$ (CMS, 2015)[2]. ESRD beneficiaries often require visits to multiple providers and follow multiple care plans, and suffer from poor health outcomes due to their underlying disease complications and multiple comorbidities. Inadequate treatment of ESRD patients can lead to high rates of hospital admission and readmission, as well as mortality rates that are higher than the general Medicare population. These alarming trends raise the need for more effective health care resource utilization to improve the quality of healthcare delivery to chronic kidney disease and ESRD patients.

In 2015, the Centers for Medicare and Medicaid Services (CMS) launched a comprehensive ESRD care (CEC) model to identify, test and evaluate new ways to improve care for Medicare beneficiaries with ESRD. The CEC model is intended to serve as a platform for CMS to partner with dialysis care providers and suppliers to test the effectiveness of a new payment and service delivery model in providing ESRD beneficiaries with patient-centered, high-quality care. The CEC model was developed in a manner similar to the Accountable Care Organizations (ACO) that were created as part of the Affordable Care Act (ACA); the first wave of 13 ESRD Seamless Care Organizations (ESCOs), participating in the Comprehensive ESRD Care Model, were identified and funded in 2015.

Dialysis centers exhibit variations in practice patterns, including choice of dialyzer reuse, whether to offer PD and late shifts, staff size and so on. Together with facility characteristics such as the geographic location, ownership type and market share, these practice patterns may potentially affect ESRD patient outcomes. Early studies of dialysis patients have examined the impact of practice patterns on patient health outcomes, such as their morality and hospitalization ratio (Held et al., 1994; Harley et al., 2013; Thomas-Hawkins et al., 2008; Yan et al., 2013; Dalal et al., 2011; Sens et al., 2011; Yang et al., 2015). Other studies have examined the nature of the relationships between clinical processes and patient outcomes (Owen et al., 1993; McClellan et al., 1998; Szczech et al., 2006; Chandrashekar et al., 2014; Held et al., 1996; Parker et al., 1994; Pisoni et al., 2004.). However, there have not been any studies on the relationship between dialysis center practice characteristics (i.e. practice patterns and facility characteristics) and clinical process measures. In this study, we aim to fill this gap in our understanding of the relationships and associations between dialysis facility practices and ESRD patient outcomes and the mediation effect of clinical processes on these relationships.

In this cross-sectional study, we draw on a recent, publicly available data set from the CMS Dialysis Facility Compare database and Medicare cost reports for free-standing dialysis providers (CMS Form 265-2011)[3]. We examine the associations between dialysis practice characteristics and process quality of care measures, related to dialysis adequacy and anemia management. Further, we study the association between these process quality measures and patient clinical outcomes, as measured by their standardized hospitalization 
ratio (SHR). We deploy seemingly unrelated regressions (SURs) to estimate a system of equations representing the associations between practice patterns, dialysis process measures and patient clinical outcomes.

We find that although dialyzer reuse is positively associated with improvements in clinical process measures, it is associated with an increase in the SHR of patients. Our results indicate that physician and nurse staffing ratios are not significantly associated with the SHR. We observe that dialysis center characteristics have significant associations with SHR. Specifically, we find that dialysis centers that are part of a larger chain/network of dialysis clinics, and those that are for-profit, are likely to exhibit higher SHR compared to independent or stand-alone dialysis centers and non-profit dialysis facilities, respectively[4]. Wealso observe that dialysis centers that have a large share of the local market are also more likely to exhibit a lower SHR, compared to smaller dialysis clinics. With respect to clinical process measures, we find that the three major dialysis process measures are significantly associated with SHR. In other words, dialysis centers with greater percentage of patients with urea reduction ratio (URR) greater than 65 per cent, and $\mathrm{Kt} / \mathrm{V}$ ratios greater than 1.2, as well as those with a lower percentage of patients with hemoglobin $(\mathrm{Hgb})$ levels less than $10 \mathrm{~g} / \mathrm{dL}$, are likely to exhibit lower hospitalization ratios. Similarly, we observe that dialysis facilities with greater proportion of adult patients receiving treatment through an arterial venous fistula (AVF) are more likely to exhibit a lower SHR.

Overall, our results indicate that dialysis facility characteristics and practice patterns are significantly associated with patient health outcomes and dialysis process quality measures. Further, our results indicate that the relationships between dialysis facility and practice characteristics and patient outcomes, measured as the SHR, are partially mediated through their impact on dialysis process quality measures. Our results have important managerial implications for operators of dialysis clinics, as they provide a better understanding of the mechanisms through which improvements in patient health outcomes are manifested.

Due to the growing movement away from volume-based payment to value-based payment models, dialysis facilities have to adjust their operations to be successful under the ESRD Prospective Payment System (PPS). Our study provides key insights on the relative cost-effectiveness of different practice patterns and provides significant managerial implications to dialysis providers to allocate their resources effectively (Bell et al., 2006).

Our study makes several major contributions. First, we address the extant gap in the literature on the relationships between dialysis facility characteristics and clinical outcomes, while specifically highlighting the role of clinical process measures as antecedents of hospitalization ratio, a key metric used to measure performance of dialysis clinics. Second, our study sheds light on the underlying mechanisms that serve as enablers of the dialysis adequacy and anemia management. To the best of our knowledge, ours is the first study to explore these relationships in the ESRD dialysis industry. Our approach provides a new direction for future studies to explore the pathways that may impact clinical quality measures in the delivery of dialysis services. By studying a relatively recent period of granular data from a large, nationally representative set of dialysis centers, our study sheds new light on the determinants of health outcomes in dialysis clinics.

\section{Background}

Dialysis is a clinical procedure that imitates the functionalities of a healthy kidney including removal of waste and excess water, keeping minerals in blood at a safe level, and controlling blood pressure. When a person loses certain kidney functionality, she is diagnosed as an ESRD patient and needs dialysis to help her survive. There are two types of dialysis: HD and PD. In HD, an external dialyzer is used externally to clean blood and wash out waste, fluid and excessive chemicals. Usually, $\mathrm{HD}$ is performed three times a week and $4 \mathrm{~h}$ in each time.
Antecedents of patient health outcomes

27 
In PD, blood is cleaned inside the patient's body through a catheter, which is placed on the belly for body access. PD is usually conducted on a daily basis. Both types of dialysis can be done in a hospital, in a dialysis center or at home. In this study, we focus on $\mathrm{HD}$ patients who receive dialysis in free-standing dialysis centers.

URR and Kt/V represent two commonly used measures of dialysis adequacy, i.e. whether dialysis removes enough urea from the blood. Kt/V consists of three elements. K represents the rate of blood passing through dialyzer, t represents unit of time, while $\mathrm{V}$ is the volume of water contained in the body. The Kt/V ratio represents the urea generated during dialysis and extra urea removed during dialysis together with fluid. The well-accepted thresholds of adequate dialysis are $\mathrm{URR} \geq 65$ per cent and $\mathrm{Kt} / \mathrm{V} \geq 1$.2. Another clinical process measure captures anemia management. As HD can cause blood loss leading to anemia, this condition tends to be serious and needs to be monitored closely for ESRD patients. The typically measure of Hgb level in blood should not be lower than $10 \mathrm{~g} / \mathrm{dL}$ for good anemia control.

Dialysis practice patterns include a wide range of processes that relate to every operational aspect of dialysis centers. Dialysis centers follow practice-specific protocols and guidelines that align with their strategic objectives, in terms of their dialysis treatment and care delivery processes. These practices may have an impact on patient outcomes and clinical performance. Earlier studies have focused on the impact of dialyzer reprocessing (i.e. reuse) on overall dialysis costs (Upadhyay et al., 2007). However, there is mixed evidence on whether cost savings associated with dialyzer reuse offset the risks of possible infection. For example, based on two years of observational data, Held et al. (1994) reported that dialyzer reuse is associated with greater mortality risk, but does not have a significant association with dialysis dosage.

During dialysis therapy, a dialysis physician or nephrologist provides kidney assessments, referrals and consultations and prescribes treatment plans, whereas a nurse treats patients and provides educational resources, encouragement and mental support. Although the impact of physician and nurse caseload has been examined frequently for other processes and conditions, few studies have studied these associations and their impacts on outcomes of dialysis patients. A recent study by Harley et al. (2013) indicates that nephrologists with high patient caseload also exhibit higher patient mortality risk, and the mortality curve plateaus as the patient-nephrologist ratio increases beyond 200. Wingard et al. (2007) conducted a concurrent study and highlighted the role of patient-focused interventions during the course of dialysis treatment. Their results showed significant decrease in mortality and hospitalization in the referral group, suggesting the effectiveness of increased nurse staffing. Thomas-Hawkins et al. (2008) observed positive relationships between patient/nurse ratio and the occurrence of nurse-reported adverse patient events, including hypotension, skipped dialysis treatments, shortened dialysis treatments and patient complaints.

The number of dialysis stations measures the capacity of a facility. The more stations that a facility has, the greater its capacity to accommodate the increasing population of dialysis patients and provide sufficient treatment sessions. We expect that the number of stations per patient to be associated with patient outcomes and clinical process measures, as too few stations may lead to negative consequences due to crowding effects, waiting times and insufficient dialysis time per patient. A recent paper by Yan et al. (2013) studied this relationship and found that smaller facilities exhibit higher mortality rates, but the mortality rate decreases as the number of dialysis stations increases. They argue that there is a threshold of 15 stations, beyond which the benefit of facility size is weakened.

The PPS incentivizes dialysis providers to select cost-efficient strategies. Although dialysis providers have begun to use PD more frequently than before, partially because 
it is a cheaper alternative to HD, the association between PD and patient outcome is unclear. On the one hand, PD patients enjoy flexible schedules, lower cost and convenience of home therapy. On the other hand, PD suffers from a few limitations such as greater risk of infection and lower accessibility compared to HD. Hirth et al. (2013) reported that the increasing use of $\mathrm{PD}$, after the introduction of bundled payments, is based on only two years of data on dialysis patients. However, other studies have shown that PD patients have higher mortality rates (Sens et al., 2011) and suffer from greater risk of early hospitalization (Yang et al., 2015). In this research, we study the impact of offering supplemental PD on patient outcomes, which provides a new perspective on the impact of this alternative model of dialysis.

The association between occurrence of late shifts and patient outcomes has mixed evidence. It is plausible that offering late shifts is beneficial to patients because it offers patients more scheduling flexibility and enables dialysis centers to extend the length of dialysis sessions. However, there has been no empirical evidence to support this perspective. In addition, the benefit of offering a late shift could be mitigated with the increasing adoption of dialysis in home setting. O'Hare et al. (2006) reported that patients in urban areas have worse survival outcomes and that dialysis facilities in these areas are more likely to offer late shifts. Their study suggests that late shifts may negatively impact patient outcomes, but a more comprehensive analysis is needed.

Compared with frequently studied associations between dialysis practice patterns and patient outcomes, relationships between practice characteristics and clinical process measures have drawn little attention. Dialysis adequacy and anemia management represent two important process measures in the Quality Incentive Program (ESRD QIP). For example, the percentage of patients with URR and Kt/V ratios greater than or equal to 65 per cent and 1.2 , respectively, are widely accepted indicators for adequate dialysis. Early studies suggest that dialyzer reuse does not significantly deteriorate patients' URR and Kt/V ratios (Ouseph et al., 1997; Garred et al., 1990; Leypoldt et al., 1998). Given the importance of clinical process measures as determinants of reimbursement, a meaningful question in dialysis effectiveness research is whether dialysis adequacy and anemia management measures are associated with practice characteristics. Our present work studies the general associations between practice patterns and clinical process measures to fill this gap and derives practical insights on the associations between process measures and dialysis outcomes.

As defined by the Agency for Healthcare Research and Quality, mortality risk and hospitalization ratio represent patient-oriented, clinical outcome measures[5]. An important question regarding dialysis operations deals with the underlying mechanisms through which patient outcomes are affected by practice patterns and the role of dialysis processes in mediating these outcomes. In other words, we are interested in studying whether the relationships between dialysis practices and facility characteristics and patient outcomes are mediated by dialysis adequacy and anemia management processes. We draw on the resource-based view of the firm to conduct an exploratory study of the relationships between dialysis facility characteristics, process measures and patient outcomes. Resource-based theory suggests that certain business process practices can provide a sustained, competitive advantage if resources are utilized effectively to develop inimitable business routines and actionable business intelligence. In the context of our study, dialysis clinics can make decisions regarding deployment of their critical resources, such as physicians and staff, as well as choice of specific practices (such as catheter usage, HD, etc). which can lead to improvements in dialysis-related clinical process measures which translate into better patient outcomes.

\section{Antecedents of patient health outcomes}




\section{Research data}

Our main data sources are the Medicare Dialysis Facility Compare (DFC) data set and Medicare cost reports for free-standing dialysis providers (CMS Form 265-2011). Medicare DFC is a public data service to facilitate the dissemination of detailed dialysis facility information about Medicare-certificated dialysis processes and to compare the quality of care provided by dialysis centers. It combines data from National Claims History Standard Analytical Files and Consolidated Renal Operations in a Web-enabled Network. From the DFC data set, we extracted data about a wide range of dialysis center characteristics and their patient profiles, including information on their chain affiliation status, ownership type, zip code, occurrence of PD and late shifts, number of dialysis stations, percentage of patients with AVF, percentage of patients with catheter left in vein longer than 90 days and several clinical process measures of dialysis adequacy and anemia management, as well as health outcomes measured as SHR.

By using a unique provider identification number, we match claims and SHR data for the same periods resulting in two years of data: January-December 2012 and January-December 2013[6]. We did not include annual data before 2012 because those data sets did not have important clinical information, such as percentage of $\mathrm{HD}$ patients with $\mathrm{Kt} / \mathrm{V} \geq 1.2$ and the percentage of patients with AVF in place. Pozniak et al. (2010) indicate that facilities with poor financial but better clinical performance, measured as having a higher percentage of $\mathrm{HD}$ patients with $\mathrm{Kt} / \mathrm{V} \geq 1.2$, are more likely to be merged and acquired by a chain (network) organization, compared to its counterparts. Because the new bundled payment system encourages facilities to make changes to their practice patterns, we focus on the data from January 2012 onward, as they provide additional insights with respect to the current state of the dialysis industry.

CMS Form 265-2011 is a public annual renal cost report starting from year 2011. It contains cost and aggregated data for free-standing dialysis centers. We extracted the number of patients, the number of total treatments, total amount of monetary expenditures, the number of nurses including contract and employment, the number of physicians including contract and employment and whether the facility reuses dialyzers to supplement the DFC data set and construct the independent variables.

We combined the two data sets by using a unique dialysis provider number. We constructed geographical location indicators for each facility by matching their zip codes with the Zip Code Tabulation Area and urban/rural information from USA Census data. After integrating these two data sets, we obtained two periods of US dialysis facility-level data for Medicare-certified free-standing facilities with 4,758 and 5,006 facility observations for 2012 and 2013, respectively.

Based on these data, we define our model variables as follows. We used the SHR to represent patient health outcome. It is calculated as the actual observed number of hospital admissions (i.e. inpatient visit) for patients at a dialysis facility divided by the expected number of admissions for the same patient, which is based on overall national rates and characteristics of these patients. The advantage of using SHR is that it adjusts for outcome-related factors, such as age, sex, diabetes, duration of ESRD, comorbidity and body mass index. Moreover, a patient is attributed to a facility only after she has received treatments from this facility for at least 60 days. This minimizes the facility-level difference in patient populations among dialysis centers, thus mitigating the drawback of using facility-level data and allowing us to conduct a facility-level study.

The practice pattern variables include Dialyzer Reuse, Nurse-To-Patient ratio, Physician-To-Patient ratio, dialysis Station-To-Patient ratio, PD and Late Shift. Dialyzer Reuse indicates whether a facility reuses dialyzers and is measured as a binary variable. 
Nurse-To-Patient ratio is defined as the number of nurses assigned to each patient and represents the average level of available nursing resources. The Physician-To-Patient and Station-To-Patient ratios are calculated as the number of physicians and dialysis stations per patient, respectively. The binary variables, $P D$ and Late Shift, indicate whether a dialysis facility offers PD and late shifts, respectively.

The clinical process measures consist of two common aspects of dialysis processes dialysis adequacy and anemia management. We use URR65 and $K t / V 1.2$ to measure the adequacy of dialysis processes, and Hgb10 to represent anemia management characteristics.

Antecedents of patient health outcomes URR65 represents the per cent of HD patients with URR $\geq 65$ per cent, whereas Kt/V1.2 means the percentage of $\mathrm{HD}$ patients with $\mathrm{Kt} / \mathrm{V} \geq 1$.2. High values of these two measures indicate more effective dialysis adequacy of the facility. The Hgb10 ratio measures percentage of $\mathrm{HD}$ patients with low Hgb levels (i.e. $\mathrm{Hgb}<10 \mathrm{~g} / \mathrm{dL}$ ) such that higher values represent a greater percentage of patients with inadequate anemia control.

We also include several controls and other measures of dialysis facility characteristics including facility chain status, ownership type, treatment volume, urban/rural indicator, cost/ patient, local market share, AVF usage and prolonged catheter usage. Ownership type (Own Type) indicates the for-profit status of a facility. Treatment volume (Treatment/Patient) measures the number of dialysis treatments per patient within one year (in 1,000 s) to capture the economy of scale. Cost/Patient is defined as the average composite cost per patient to capture the expenditure efficiency. Local Market Share measures the percentage of patients cared for in a dialysis facility relative to total number of patients who live in the three-digit zip code, a measure of local market concentration[7]. We use the percentage of patients with AVF (AVF Usage) and the per cent of patients with vascular catheter in use for 90 days or longer (Catheter Usage) as proxies to measure vascular access performance measures. Higher values of AVF and lower value of long-term catheter use indicate that more patients received appropriate and superior vascular access.

We note that missing values do exist in the hospitalization ratio and clinical process variables. The number of observations with missing values is less than 5 per cent of our overall data (190 and 213 for 2012 and 2013 data, respectively). For a majority of observations with missing values, Medicare categorizes the reasons as either the number of patients is too small to report or the facility is not open for the entire reporting period. We deployed a list-wise deletion strategy to remove observations with missing values. (Allison, 2001).

Our variable definition and sample descriptive statistics are included in Tables I and II, respectively. Table III presents the correlations across the model variables in our study.

\section{Empirical approach}

Given the two-year panel data set, we use one-year lagged predictors for all analyses to address the potential endogeneity issue. For example, hiring more clinical staff, such as physicians and nurses, could impact clinical processes as well as patient outcomes. Conversely, a dialysis facility might decide to hire more clinical staff because the percentage of its patients with adequate dialysis is low. We mitigate the effect of reverse causality which may bias our estimation results, using lagged independent variable models. However, this may result in patient population mismatch for each facility because patients may sometimes change their attending facilities and our data set captures aggregated facility characteristics instead of patient-level determinants. Dialysis Patient Citizens (2012) reported that only 2 per cent of patients were highly unsatisfied with their care and nearly 80 per cent respondents were highly satisfied in its 2012 patient survey[8]. Therefore, we believe that the percentage of patients who switch their dialysis providers is small and our results are qualitatively robust. State dummies are included in all models to control for state-specific heterogeneity, 
Patient outcome

SHR

Clinical processes

URR65

$\mathrm{Kt} / \mathrm{V} 1.2$

Hgb10

Facility characteristics

Chain Type

Own Type

Treatment/Patient

Urban

Cost/Patient

Local Market Share

Practice pattern

AVF Usage

Catheter Usage

Dialyzer Reuse

Nurse-To-Patient ratio

Physician-To-Patient ratio

Station-To-Patient ratio

PD

Late Shift
Standardized Hospitalization Ratio, calculated as the actual observed no. of hospitalization admissions for the patients at a facility divided by the expected no. of admissions for the same patients after risk adjusted

Indicates the percentage of patients who had URR greater than or equal to 65 per cent

Indicates the percentage of adult $\mathrm{HD}$ patients with $\mathrm{Kt} / \mathrm{V}$ greater than or equal to 1.2

Indicates the percentage of patients who had average Hgb less than $10.0 \mathrm{~g} / \mathrm{dL}$

Indicates a facility's affiliation status; $1=$ affiliate to a chain, $0=$ otherwise

Indicates a facility's for-profit status; 1 = for-profit, $0=$ not for-profit Indicates the number of dialysis treatments offered per patient Indicates a facility's geographic location; $1=$ urban, $0=$ rural Indicates the average composite cost per treatment in US\$1,000 Indicates the market concentration in terms of patient number at the 3-digit-level zip code

Indicates the percentage of adult patients who received treatment through an arterial venous fistula (AVF)

Indicates the percentage of adult patients who had a catheter (tube) left in a vein longer than 90 days for their regular $\mathrm{HD}$ treatments Indicates whether a facility reuses dialyzers; $1=$ reuse, $0=$ no reuse Indicates the number of nurses per patient

Indicates the number of physicians per patient Indicates the number of dialysis stations per patient

Indicates whether a facility offers in-center peritoneal dialysis (PD); $1=$ offer, $0=$ no offer

Indicates whether or not the facility has a shift starting at 5:00 p.m. or later; $1=$ offer, $0=$ no offer
Table I.

Variable definition

Notes: SHR is from Y2013, while other facility characteristics and practice patterns are from Y2012. Both periods of URR65, Kt/V1.2 and Hgb10 are used

such as various consumption price levels across states. Our results are qualitatively consistent when we use network dummies instead of state dummies. Our analysis of relations between facility practice patterns and clinical processes is based on estimation of the following system of equations:

$$
\begin{aligned}
\text { Clinical ProcessMeasure }_{i t}= & \beta_{0}+\beta_{1} \text { AVFUsage }_{i t-1} \\
& +\beta_{2} \text { CatheterUsage }_{i t-1}+\beta_{3} \text { DialyzerReuse }_{i t-1} \\
& +\beta_{4} \text { NurseToPatientRatio }_{i t-1} \\
& +\beta_{5} \text { PhysicianToPatientRatio }_{i t-1} \\
& +\beta_{6} \text { StationToPatientRatio }_{i t-1} \\
& +\beta_{7} \text { PD }_{i t-1}+\beta_{8} \text { LateShift }_{i t-1}+\gamma X_{i t-1}+S_{i}+\varepsilon_{i}
\end{aligned}
$$




\begin{tabular}{|c|c|c|c|c|c|c|}
\hline Practice characteristic & Mean & $\mathrm{SD}$ & Minimum & Maximum & Obs & Antecedents of \\
\hline $\begin{array}{l}\text { Patient outcome } \\
\text { SHR }\end{array}$ & 1.0008 & 0.2922 & 0.15 & 2.7 & 4591 & outcomes \\
\hline $\begin{array}{l}\text { Clinical performance Y2012 } \\
\text { URR65 } \\
\text { Kt/V1.2 } \\
\text { Hgb10 }\end{array}$ & $\begin{array}{l}98.6166 \\
88.2783 \\
10.318\end{array}$ & $\begin{array}{l}2.5606 \\
8.9278 \\
9.5418\end{array}$ & $\begin{array}{r}70 \\
0 \\
0\end{array}$ & $\begin{array}{r}100 \\
100 \\
91\end{array}$ & $\begin{array}{l}4483 \\
4556 \\
4466\end{array}$ & 33 \\
\hline $\begin{array}{l}\text { Clinical performance Y2013 } \\
\text { URR65 } \\
\text { Kt/V1.2 } \\
\text { Hgb10 }\end{array}$ & $\begin{array}{l}98.8413 \\
88.9614 \\
12.4882\end{array}$ & $\begin{array}{l}2.1933 \\
7.8655 \\
9.9284\end{array}$ & $\begin{array}{r}72 \\
0 \\
0\end{array}$ & $\begin{array}{r}100 \\
100 \\
75\end{array}$ & $\begin{array}{l}4500 \\
4557 \\
4486\end{array}$ & \\
\hline $\begin{array}{l}\text { Facility characteristics } \\
\text { Chain Type } \\
\text { Own Type } \\
\text { Treatment/Patient } \\
\text { Urban } \\
\text { Cost/Patient } \\
\text { Local Market Share }\end{array}$ & $\begin{array}{c}0.9068 \\
0.9226 \\
147.989 \\
0.9783 \\
34.0098 \\
0.1573\end{array}$ & $\begin{array}{r}0.2908 \\
0.2672 \\
149.6725 \\
0.1458 \\
37.9303 \\
0.1959\end{array}$ & $\begin{array}{l}0 \\
0 \\
0.7826 \\
0 \\
6.9571 \\
0.0003\end{array}$ & $\begin{array}{c}1 \\
1 \\
8419 \\
1 \\
2067.565 \\
1\end{array}$ & $\begin{array}{l}4602 \\
4601 \\
4602 \\
4602 \\
4602 \\
4602\end{array}$ & \\
\hline $\begin{array}{l}\text { Practice pattern } \\
\text { AVF Usage } \\
\text { Catheter Usage } \\
\text { Dialyzer Reuse } \\
\text { Nurse-To-Patient ratio } \\
\text { Physician-To-Patient ratio } \\
\text { Station-To-Patient ratio } \\
\text { PD } \\
\text { Late Shift }\end{array}$ & $\begin{array}{r}61.2488 \\
11.1089 \\
0.4344 \\
0.0726 \\
0.0039 \\
0.3218 \\
0.4694 \\
0.196\end{array}$ & $\begin{array}{l}11.1387 \\
6.491 \\
0.4957 \\
0.0899 \\
0.0219 \\
0.32 \\
0.4991 \\
0.397\end{array}$ & $\begin{array}{r}15 \\
0 \\
0 \\
0 \\
0 \\
0 \\
0 \\
0\end{array}$ & $\begin{array}{l}100 \\
56 \\
1 \\
3.58 \\
0.6667 \\
16 \\
1 \\
1\end{array}$ & $\begin{array}{l}4574 \\
4574 \\
4602 \\
4602 \\
4602 \\
4602 \\
4602 \\
4602\end{array}$ & \\
\hline
\end{tabular}

Notes: SHR is from 2013, while other facility characteristics and practice patterns are from 2012. Clinical processes Y2012 and Y2013 indicate URR65, Kt/V1.2 and Hgb10 data from 2012 and 2013, respectively

Table II.

Descriptive statistics

where Clinical Process Measures $_{i t}$ include URR65, Kt/V1.2 and Hgb10. The variables that represent practice patterns are AVF Usage, Catheter Usage, Dialyzer Reuse, Nurse-To-Patient ratio, Physician-To-Patient ratio, Station-To-Patient ratio, PD and Late Shift[9]. The vector $X$ represents control variables that measure dialysis facility characteristics including facility chain status, ownership type, treatment volume per patient, urban/rural status, treatment cost per patient and local market share. The vector $S$ represents state-specific dummies. We first estimate the above system of equations using ordinary least squares (OLS) regressions with White robust standard errors.

In the ESRD QIP, the clinical process measures consist of URR $\geq 65$ per cent, $\mathrm{Hgb}<10$ $\mathrm{g} / \mathrm{dL}$ and $\mathrm{Hgb}>12 \mathrm{~g} / \mathrm{dL}$ in payment years 2012 and 2013[10]. To avoid reductions in payments, all dialysis facilities have economic incentives to exert sufficient efforts to meet the benchmarks for these measures. In addition, because URR $\geq 65$ per cent is a high benchmark relative to $\mathrm{Kt} / \mathrm{V} \geq 1.2$, a dialysis facility which endeavors to improve its average URR ratio will also attempt to increase its average $\mathrm{Kt} / \mathrm{V}$. With respect to these considerations, there is a high probability that correlations exist across error terms for the three equations represented in the system of equations shown in equation (1). In such situations, equation-by-equation OLS still offers consistent estimates, even though the 
JCC
10,1

34

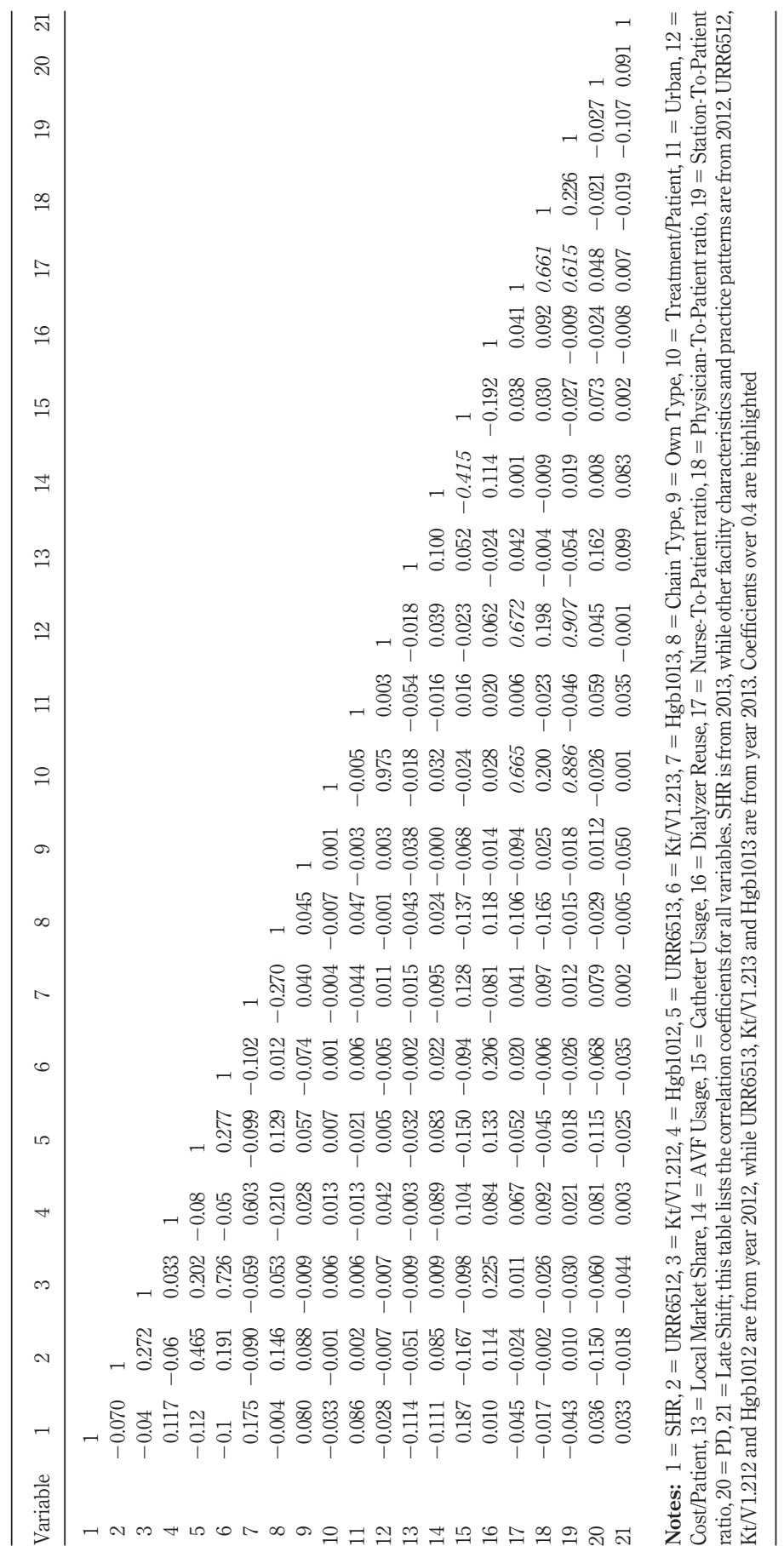

Table III.

Variable correlation 
SUR model provides more efficient estimates by using the Generalized Least Square (GLS) estimator.

As the SUR estimator is exactly the same as an OLS estimator, when the right-hand side variables are the same across equations, we deliberately drop insignificant variables from each equation, based on the OLS results. We then use the SUR model to estimate the system of equations below:

$$
\begin{aligned}
& \text { URR65 }_{i t}=\beta_{0}+\beta_{1} \text { CatheterUsage }_{i t-1}+\beta_{2} \text { DialyzerReuse }_{i t-1} \\
& +\beta_{3} \text { NurseToPatientRatio }_{i t-1}+\beta_{4} \text { PhysicianToPatientRatio }_{i t-1} \\
& +\beta_{5} \text { StationToPatientRatio }_{i t-1}+\beta_{6} P D_{i t-1}+\gamma_{1} \text { ChainType }_{i t-1} \\
& +\gamma_{2} \text { OwnType }_{i t-1}+\gamma_{3} \text { Treatment/Patient }_{i t-1}+\gamma_{4} \text { Urban }_{i t-1} \\
& +\gamma_{5} \text { Cost/Patient }_{i t-1}+S_{i}+\varepsilon_{i} \\
& K t / V 1.2_{i t}=\beta_{0}+\beta_{1} \text { CatheterUsage }_{i t-1}+\beta_{2} \text { DialyzerReuse }_{i t-1} \\
& +\beta_{3} \text { NurseToPatientRatio }{ }_{i t-1}+\beta_{4} \text { PhysicianToPatientRatio }_{i t-1} \\
& +\beta_{5} \text { StationToPatientRatio }_{i t-1}+\beta_{6} P_{i t-1}+\beta_{7} \text { LateShift }_{i t-1} \\
& +\gamma_{1} \text { ChainType }_{i t-1}+\gamma_{2} \text { OwnType }_{i t-1}+\gamma_{3} \text { Treatment/Patient }_{i t-1} \\
& +\gamma_{4} \text { Urban }_{i t-1}+\gamma_{5} \text { Cost/Patient }_{i t-1}+S_{i}+\varepsilon_{i} \\
& \text { Hgbl0 }_{i t}=\beta_{0}+\beta_{1} \text { AV FUsage } \text { it }-1+\beta_{2} \text { CatheterUsage }_{i t-1} \\
& +\beta_{3} \text { DialyzerReuse }_{i t-1}+\beta_{4} \text { Nurse ToPatientRatio }_{i t-1} \\
& +\beta_{5} \text { PhysicianToPatientRatio }_{i t-1}+\beta_{6} \text { StationToPatientRatio }_{i t-1} \\
& +\beta_{7} P D_{i t-1}+\gamma_{1} \text { ChainType }_{i t-1}+\gamma_{2} \text { OwnType }_{i t-1} \\
& +\gamma_{3} \text { Treatment/Patient }_{i t-1}+\gamma_{4} \text { Urban }_{i t-1} \\
& +\gamma_{5} \text { Cost/Patient }_{i t-1}+\gamma_{6} \text { LocalMarketShare }_{i t-1}+S_{i}+\varepsilon_{i}
\end{aligned}
$$

where we drop Local Market Share, AVF Usage and Late Shift from equation (1A); Local Market Share and AVF Usage from equation (1B); and Late Shift from equation (1C). Both White and Breusch-Pagan tests, in our preliminary estimation of equation (1), support the existence of heteroscedasticity $(\phi<0.001$ and $p<0.001$, respectively). Hence, we adopt the generalized SUR approach described in Greene (2003). We first transform the data by using OLS residuals from a preliminary OLS analysis. To derive the format of transformation function, we use the idea of GLS suggested in Wooldridge (2012), and assume the error term is of the general format shown as:

$$
\operatorname{Var}\left(u_{i} \mid Z_{i}\right)=\sigma_{i}^{2} \exp \left(Z_{i} \delta_{i}\right), \quad i \in\{A, B, C\}
$$

where $Z_{i}$ is the matrix of independent variables in equation $i$, and $\delta_{i}$ is the matrix of parameters in corresponding equations. After the transformation of equations $(1 \mathrm{~A}),(1 \mathrm{~B})$ and (1C), the system of equations is estimated by GLS to study the associations between the three clinical process measures and our dialysis practice variables. The estimate results are reported in Table IV. We observe that SUR and OLS estimation results are qualitatively consistent in terms of the sign and significance of the estimated coefficients.

Antecedents of patient health outcomes 


\section{JCC \\ 10,1}

Table IV.

SUR estimations of clinical process measures

$\begin{array}{llll}\text { Model variable } & \text { URR65 } & \text { Kt/V1.2 } & \text { Hgb10 }\end{array}$

Facility characteristics

Chain Type

$0.693 * * *(0.140)$

Own Type

$0.492 * * *(0.136)$

Treatment/Patient

$-0.002(0.001)$

$-0.197(0.155)$

$0.0003(0.005)$

$$
\begin{array}{r}
-0.036(0.429) \\
-1.443 * * *(0.359) \\
0.014 * * *(0.003) \\
-0.698(0.669) \\
-0.070 * * *(0.016)
\end{array}
$$

$-8.160 * * * * 0.722)$

$1.320 * * *(0.444)$

$-0.023 * * *(0.004)$

$0.582(1.178)$

$0.141 * * *(0.019)$

$-1.220 *(0.632)$

Local Market Share

Practice pattern

AVF Usage

Catheter Usage

Dialyzer Reuse

Nurse-To-Patient ratio

Physician-To-Patient ratio

$-0.020 * * *(0.005)$

$0.341 * * *(0.058)$

$-0.754(0.911)$

$-5.593(4.160)$

Station-To-Patient ratio

$1.012 * * *(0.246)$

PD

$-0.203^{* * *}(0.060)$

Late Shift

State Dummies

Obs

Yes

4444

$$
\begin{gathered}
-0.059 * * *(0.016) \\
3.233 * * *(0.204) \\
5.563 *(3.075) \\
-1.670(18.276) \\
0.146(0.943) \\
-0.261(0.200) \\
-0.440 *(0.232) \\
\text { Yes } \\
4444
\end{gathered}
$$

Notes: Significantly different from 0 at: $* p<0.1 ; * * p<0.05$; *** $p<0.01$. This table includes regressions using dependent variable from 2013 and independent variables from 2012. Local Market Share is calculated based on same first three digits. Transformations are made according to general weight format denoted in Wooldridge (2012) to deal with potential heteroscedasticity. One outlier observation is dropped deliberately to avoid collinearity

Next, we estimate the associations between dialysis practice patterns, facility characteristics and patient health outcomes, measured using SHR. We estimate the following system of hierarchical linear regressions, to separately estimate the specific associations between dialysis practice characteristics and clinical process measures:

$$
\begin{aligned}
& \text { SHR }_{i t}=\beta_{0}+\beta_{1} \text { DialyzerReuse }_{i t-1}+\beta_{2} \text { NurseToPatientRatio }_{i t-1} \\
& +\beta_{3} \text { PhysicianToPatientRatio }_{i t-1}+\beta_{4} \text { StationToPatientRatio }_{i t-1} \\
& +\beta_{5} P D_{i t-1}+\beta_{6} \text { LateShift }_{i t-1}+\gamma X_{i t-1}+S_{i}+\varepsilon_{i} \\
& S H R_{i t}=\beta_{0}+\delta_{1} \text { URR65 }_{i t-1}+\delta_{2} K t / V 1.2_{i t-1}+\delta_{3} H g b 10_{i t-1}+S_{i}+\varepsilon_{i} \\
& \text { SHR }_{i t}=\beta_{0}+\beta_{1} \text { DialyzerReuse }_{i t-1}+\beta_{2} \text { NurseToPatientRatio }_{i t-1} \\
& +\beta_{3} \text { PhysicianToPatientRatio }_{i t-1}+\beta_{4} \text { StationToPatientRatio }_{i t-1} \\
& +\beta_{5} P D_{i t-1}+\beta_{6} \text { LateShift }_{i t-1}+\delta_{1} \text { URR65 }_{i t-1}+\delta_{2} \text { Kt/V1.2 } 2_{i t-1} \\
& +\delta_{3} H g b 10_{i t-1}+\gamma X_{i t-1}+S_{i}+\varepsilon_{i}
\end{aligned}
$$

We follow the approach described by Baron and Kenny (1986) to examine the mediation effects of clinical processes. First, we estimate the direct effects of practice patterns on SHR using equation (2A). Next, we estimate equation (2B) to examine the effects of the intermediate clinical processes on SHR. Finally, we examine the overall effects of practice patterns on SHR after accounting for the effects of clinical processes through equation 
(2C). By comparing our estimates across these three equations, we are able to study whether the impacts of practice patterns and facility characteristics on SHR are mediated through clinical processes. We summarize these relationships in our conceptual research model as shown in Figure 1. For a significant mediation effect, an intervention must be significantly associated with SHR and the dialysis process measures, but its association with SHR needs to be either completely or partially explained away. The former scenario, where the direct association between the independent variables and SHR is statistically insignificant, is referred as the full mediation model, while the latter scenario, where the magnitude of the effect is reduced, is known as partial mediation. The estimation results of equation (2C) allow us to test the significance of the full versus partial medication effect.

The correlation matrix of the model variables, as presented in Table III, indicates that the correlations between the independent variables are generally below 0.4 and do not indicate the presence of multicollinearity. Furthermore, the Variance Inflation Factors (VIFs) of major variables are below 5 , confirming the absence of multicollinearity among our variables of interest. As the White and Breusch-Pagan tests suggest the existence of heteroscedasticity $(p<0.001$ and $p<0.001$, respectively), we estimate these equations using OLS with White robust standard errors.

\section{Results}

\subsection{Practice pattern and clinical process measures}

Table IV displays the results of SUR estimations of the three clinical process measures against practice patterns with facility characteristics as controls. Columns 1 and 2 specify the regressions using URR65 and Kt/V1.2 dialysis process measures as dependent variables, respectively, whereas Column 3 reports the result of the regression using $\mathrm{Hgb10}$ as the dependent variable. All three regressions control for state-specific heterogeneity.

The results in Table IV suggest that dialysis facilities that are part of larger networks or chains are likely to exhibit a 0.69 per cent higher percentage of patients with URR $\geq 65$ per cent, but 8.16 per cent lower percentage of patients with $\mathrm{Hgb}<10 \mathrm{~g} / \mathrm{dL}$, compared to facilities that are not part of a chain. These coefficients are significant at the 1 per cent level and qualitatively consistent with the results reported by Zhang et al. (2011) in the sense that affiliated dialysis centers tend to exhibit desirable clinical performance. The coefficients of ownership type show that for-profit facilities have a 0.49 per cent higher percentage of patients with URR $\geq 65$ per cent, but 1.44 per cent lower percentage of patients with $\mathrm{Kt} / \mathrm{V} \geq 1.2$, compared to non-profit facilities. Compared to URR ratio, Kt/V

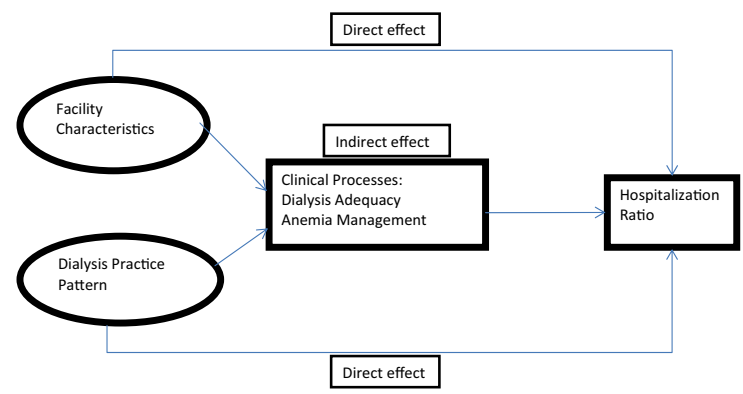

Antecedents of patient health outcomes 
captures the amount of urea removed with excess fluid and is recognized to be a more accurate measure of dialysis effectiveness[11]. Therefore, for-profit facilities tend to have worse clinical process measures, i.e. lower percentage of patients with adequate dialysis (Kt/V1.2 ratio) and 1.32 per cent higher percentage of patients with low Hgb level.

The estimates of treatment volume per patient indicate that when one additional treatment is provided to each patient, the percentage of patients with $\mathrm{Kt} / \mathrm{V} \geq 1.2$ will be 0.01 per cent higher and the per cent with $\mathrm{Hgb}<10 \mathrm{~g} / \mathrm{dL}$ will be 0.02 per cent lower, on average. This result suggests that an increase in the number of treatments can benefit the dialysis facility by reducing the average cost and utilizing dialysis machines more efficiently, which can enable more effective clinical processes. The treatment cost per patient measures the efficiency of expenditure allocation, holding other variables constant. The results indicate that a $\$ 1,000$ increase in average cost per dialysis session is associated with 0.07 per cent reduction in patient $\mathrm{Kt} / \mathrm{V}$ ratio, and 0.14 per cent increase in patients with low Hgb level. The coefficients of cost/patient suggest that greater expenditures on patients alone are not necessarily associated with better dialysis process outcomes. It is possible that the patient severity (or case mix index) may increase the costs expended per patient even though their dialysis process measures are lower than average. The coefficient of local market share is significant and shows that 1 per cent increase in market share is associated with 0.01 per cent fewer patients with Hgb $<10 \mathrm{~g} / \mathrm{dL}$. Overall, our results indicate significant associations between clinical process performances and dialysis center characteristics in terms of chain type, own type, treatment volume, treatment cost and market occupation.

In terms of practice patterns, the coefficients of AVF Usage and Catheter usage are expected. With a 1 per cent increase in patients with AVF, a facility has around 0.05 per cent fewer patients with $\mathrm{Hgb}<10 \mathrm{~g} / \mathrm{dL}$. When a facility reduces prolonged usage of catheters by 1 per cent, 0.02 per cent and 0.06 per cent more patients will have adequate dialysis measures in terms of their URR and Kt/V ratios, respectively, and about 0.07 per cent fewer patients exhibit low Hgb risk. The coefficients of dialyzer reuse are interesting in that a facility with dialyzer reuse has 0.34 per cent more patients with URR $\geq 65$ per cent, 3.23 per cent more with $\mathrm{Kt} / \mathrm{V} \geq 1.2$ and 0.93 per cent fewer patients with $\mathrm{Hgb}<10 \mathrm{~g} / \mathrm{dL}$, compared to facilities which do not reuse dialyzers. From a clinical perspective, reuse of dialyzers could be beneficial to patients and dialysis centers by saving time and money. Previous research suggests that dialyzer reuse shows either a small or no additional risk compared to single-use dialyzer (Lacson and Lazarus, 2006; Twardowski, 2006). With strict adherence to certain standards, dialyzers can be reused safely. The benefits are substantial, as a facility can afford to utilize more sufficient dialysis sessions and provide higher dose of dialysis with the resultant cost savings. Therefore, our results suggest that the benefits dominate and dialyzer reuse contributes to desirable dialysis adequacy and anemia control levels.

We find that greater nurse-to-patient ratio is not significantly associated with the dialysis adequacy measure of URR and Hgb levels. The coefficient of nurse-to-patient ratio is marginally significant in the Kt/V1.2 regression, suggesting that 10 units of increase in the nurse-to-patient ratio is associated with 0.31 per cent higher percentage of patients with $\mathrm{Kt} / \mathrm{V}$ $\geq 1.2[12]$. With more nurses assigned to each patient, patients may accrue the benefits of sufficient educational resources, scrutinized medication management and flexible schedules. Our result of nurse staffing being associated with better clinical process measures is consistent with early results in a range of domains regarding the impact of nurse staffing (Pronovost et al., 2001). 
The results for physician-to-patient staffing ratio suggest that it is not significantly associated with dialysis adequacy process measures, i.e. URR65 and Kt/V1.2. However, its positive coefficient in Column 3 of Table IV suggests that an increase of 10 units in the physician-to-patient ratio is associated with an increase of 0.08 per cent in patients with low Hgb level[13]. Although the effect of physician-to-patient ratio is relatively small, we observe that almost half of free-standing dialysis facilities do not have any physicians on site, which supports our estimation results that employing more physicians hurts patients' Hgb control process measure. As we study free-standing dialysis centers exclusively, a majority of which are small facilities with less than 100 patients, employing a physician brings limited benefits. Therefore, the cost per patient for physicians employed is high and may force the facility to compromise other aspects of services which outweigh the benefit of hiring physicians, thereby resulting in a negative association with anemia management.

The coefficients of station-to-patient ratio are significant in Columns 1 and 3[14]. The result reveals that a one unit increase in station-to-patient ratio is associated with 0.17 per cent more patients with URR $\geq 65$ per cent and 0.90 per cent lower per cent of patients with $\mathrm{Hgb}<10 \mathrm{~g} / \mathrm{dL}$. In other words, by increasing the number of dialysis stations in a dialysis clinic, patients can extend their dialysis sessions to receive more adequate sessions and have greater scheduling flexibility with respect to available stations. These benefits are observed in terms of greater adherence with respect to the adequacy of dialysis and anemia levels.

To complement $\mathrm{HD}$ and meet the requirements of ESRD patients, some facilities also offer PD treatment. The coefficients of PD indicate that a facility offering PD modality has 0.20 per cent fewer patients with URR $\geq 65$ per cent and 0.87 per cent with $\mathrm{Hgb}<10 \mathrm{~g} / \mathrm{d}$, compared to a facility that does not provide PD. Although PD costs are significantly less than HD, patients receiving PD are more likely to have high infection rates of peritonitis, especially in a home setting. From a dialysis facility's perspective, setting up PD incurs the cost of hiring a PD specialist, training staff for providing PD and purchasing supporting equipment. These concerns related to offering PD dominate the cost benefit, thus impairing improvements to the clinical process measures. Offering late night shift is associated with 0.44 per cent lower percentage of patients with $\mathrm{Kt} / \mathrm{V} \geq 1.2$. One would expect that offering late shift extends the flexibility for patients with special scheduling needs who are willing to come at night. However, it also may increase process complexity related to hiring of proper staff who are willing to work night shifts and might deteriorate employee satisfaction due to work stress and increase the probability of medical errors during the late shift. The results support our argument that dialysis practice patterns are significantly related to clinical process measures.

\subsection{Determinants of hospitalization ratio}

Next, we study the association between dialysis facility characteristics and practice patterns and their SHR, as shown in Table V. Our model fit is generally quite high, with $R^{2}$ values ranging from 0.18 to 0.22 , with statistically significant values of the corresponding $F$-statistics. In Column 1, we report the OLS results of the "direct effect" model, as shown in equation (2A), where we estimate the direct associations between practice patterns and facility characteristics and SHR. We note that dialysis facilities that are for-profit entities are likely to exhibit 7.29 per cent higher SHR (coeff. = 0.0729; $p<0.01$ ), compared to non-profit dialysis clinics. We also observe that the number of treatments per patient (Treatment/patient) is negatively associated with SHR (coeff. = $-0.0003 ; p<0.05$ ), which suggests that dialysis facilities with greater treatment per 


\section{JCC \\ 10,1}

40

Table V.

OLS estimation results for SHR
Model variable

(1)

(2)

(3)

\section{Facility characteristics}

Chain Type

Own Type

Treatment/Patient

Urban

Cost/Patient

Local Market Share

$$
\begin{gathered}
0.002(0.017) \\
0.073^{* * *}(0.015) \\
-0.0003^{* *}(0.0001) \\
-0.057 *(0.032) \\
0.002^{* *}(0.001) \\
-0.173^{* * *}(0.023)
\end{gathered}
$$

$$
\begin{array}{r}
0.033 * *(0.017) \\
0.071^{* * * *}(0.015) \\
-0.023^{* * *}(0.004) \\
0.050(0.032) \\
0.001(0.001) \\
-0.174^{* * * *}(0.022)
\end{array}
$$

\section{Clinical Performance}

URR65

$\mathrm{Kt} / \mathrm{V} 1.2$

Hgb 10

$$
\begin{aligned}
& -0.006^{* * * *}(0.002) \\
& -0.001 * * *(0.001) \\
& -0.004^{* * * *}(0.001)
\end{aligned}
$$

$-0.006^{* * * *}(0.002)$

$-0.001^{* *}(0.001)$

$-0.004^{* * * *}(0.001)$

\section{Practice pattern}

\section{AVF Usage}

Catheter Usage

Dialyzer Reuse

$-0.001^{* *}(0.001)$

$0.005 * * *(0.001)$

$0.008(0.009)$

Nurse-To-Patient ratio

Physician-To-Patient ratio

$-0.043(0.071)$

$-0.143(0.314)$

$0.071 * *(0.032)$

Station-To-Patient ratio

PD

$-0.011(0.009)$

$0.023^{* *}(0.010)$

State
4571
0.194

$$
\begin{gathered}
-0.001 * *(0.0004) \\
0.005 * * *(0.001) \\
0.016 *(0.009) \\
-0.018(0.087) \\
0.266(0.384) \\
-0.061(0.039) \\
0.011(0.009) \\
0.021 * *(0.010) \\
\text { State } \\
4434 \\
0.218
\end{gathered}
$$

Obs

$R^{2}$
4435

0.186

Notes: Significantly different from 0 at: $* p<0.1 ; * * p<0.05 ; * * * p<0.01$. This table includes regressions using dependent variable (SHR) from 2013 and independent variables from 2012. Local Market Share is calculated based on same first three digits. Each model is estimated using equation-by-equation OLS. White robust standard errors are presented in parentheses

patient ratios are likely to exhibit lower SHR. We also observe that dialysis clinics that have a larger share of the local market are likely to exhibit a lower SHR. Specifically, a 1 per cent increase in local market share is associated with a 0.17 per cent decrease in the average SHR of the corresponding dialysis clinic. We also observe that dialysis clinics that are located in urban areas exhibit 5.73 per cent higher incidence of hospitalization. Dialysis centers that incur $\$ 1,000$ higher cost-per-patient are likely to have 0.15 per cent more hospitalizations, which may be partly explained due to the possibility of such clinics also admitting more severely ill patients. These results confirm the existence of direct effects of facility characteristics on patient outcome.

With respect to practice patterns, we observe that a 1 per cent increase in the use of AVF is associated with a 0.09 per cent reduction in SHR. Similarly, a 1 per cent increase in the station-to-patient ratio is also associated with a 0.07 per cent reduction in SHR. On the other hand, we also observe that a 1 per cent increase in the percentage of patients on prolonged catheter usage is associated with 0.51 per cent higher risk of hospitalization. Our results are consistent with previous studies which reported that prolonged use of catheters is more likely to cause patient deaths due to the greater risk of infections (Hollenbeck et al., 2012). We note that dialysis clinics that offer late shifts are also likely to report greater SHR, compared to those that do not. Specifically, dialysis facilities that have late shift schedules have 2.33 per cent higher SHR than facilities that do not offer 
late shift. Other practice patterns, such as dialyzer reuse, PD and nurse-to-patient and physician-to-patient ratios, are not statistically significant in terms of their association with SHR. Overall, our results support the direct effects of practice patterns on hospitalization ratio.

In Column 2 of Table $\mathrm{V}$, we report the estimation results for the complete mediation model as specified in equation (2B). Specifically, we report the regression coefficients for the three dialysis process measures and evaluate their associations with SHR. We observe that a 1 per cent increase in the percentage of patients with URR $\geq 65$ per cent is associated with a 0.61

Antecedents of patient health outcomes per cent reduction in SHR. Similarly, a 1 per cent increase in the Kt/V ratio is associated with a 0.14 per cent drop in the SHR of a dialysis clinic. In a similar manner, we report that a 1 per cent reduction in the percentage of patients with low Hgb levels (i.e. Hgb $<10 \mathrm{~g} / \mathrm{dL}$ ) is associated with a corresponding decrease of 0.40 per cent in the SHR. Hence, we observe that improvements in the two process measures of dialysis adequacy (URR $\geq 65$ per cent and $\mathrm{Kt} / \mathrm{V} \geq 1.2)$ and the measure of anemia management $(\mathrm{Hgb}<10 \mathrm{~g} / \mathrm{dL})$ are associated with corresponding reduction in SHR. We observe that the complete mediation model explains 18.63 per cent of the variation in SHR, compared to the "direct effects" model which shows that dialysis facility characteristics and practice patterns account for 19.42 per cent of the variation in SHR.

Finally, we present the estimation results of the "full" model, specified in equation (2C), as shown in Column 3 of Table V. First, we observe that the coefficients of the three dialysis process measures remain statistically significant and consistent with earlier results presented in Column 2. We also note the percentage of variation explained by the predictors in the "full" model is 21.78 per cent, a marginal increase from the variation explained by the "direct effects" and "complete mediation" models. Although most of the coefficients of the facility characteristics and practice patterns remain qualitatively similar to the results presented in Column 1, in terms of their signs and significance, there are some salient differences.

We observe that dialysis facilities that belong to a chain or network are more likely to exhibit higher SHR (coeff. $=0.033, p<0.01$ ). In other words, a networked dialysis center is likely to exhibit a SHR that is 3.3 per cent higher than an independent dialysis center that is not part of a larger network, ceteris paribus. The coefficients of Own_type and local market share are similar to the earlier results in Column 1 . They indicate that for-profit dialysis centers are likely to exhibit a 7.07 per cent higher SHR compared to their non-profit counterparts, and that facilities that have 1 per cent high market share are likely to exhibit 0.17 per cent lower hospitalization ratio. The coefficients of $A V F$ Usage, Catheter Usage and Late Shift are also qualitatively similar to their estimated values reported in Column 1. To be precise, a 1 per cent increase in patients using AVF is associated with 0.11 per cent reduction in SHR, while 1 per cent more patients with catheter usage is related to 0.46 per cent higher hospitalization ratio. With respect to late shift schedule, dialysis centers with late shifts have 2.12 per cent more patient hospitalizations compared to facilities which do not offer late shifts. We observe that the coefficient of Dialyze Reuse is marginally significant at a $p$-value $<0.10$, which indicates that if a facility reuses dialyzers, its patients exhibit a 1.57 per cent higher hospitalization risk. When combined with the earlier results reported in Table IV, these results suggest that although dialyzer reuse is beneficial for clinical processes because of cost savings, it is not desirable in terms of the greater hospitalization risk. The main advantage of dialyzer reuse is economic. This result is also consistent with prior studies which have reported the adverse consequences of dialyzer reuse (Port et al., 2001; Feldman et al., 1999; Lowrie et al., 2004). 
We also note that nurse-to-patient ratio, physician-to-patient ratio, station-to-patient ratio and $\mathrm{PD}$ are not significantly associated with SHR. On the one hand, with more nurses assigned to each patient, patients can enjoy a higher quality of clinical care as manifested through improvements in clinical process measures. On the other hand, Aiken et al. (2002) suggest the dialysis facilities need to provide sufficient support to nurses to generate positive impact on patient outcomes. If adequate support is not provided to nurses, increasing the nurse-to-patient ratio may not translate into better patient health outcomes.

Our results also indicate that physician caseload and station caseload are not significantly associated with SHR in our analysis. A deeper analysis shows that the average physician-to-patient ratio is 0.0039 in our data set, or equivalently the physician-to-patient ratio is 1:256. At the 75 per cent threshold, the physician caseload is 260. Therefore, a majority of the dialysis facilities have caseloads that are higher than the physician caseload threshold. On average, each facility has over 18 stations and 70 per cent of facilities have at least 15 stations. Similarly, Yan et al. (2013) argue that the benefit of facility size is weak for the majority of observations. Therefore, our results are consistent with earlier studies with respect to the lack of significance of the station and physician staffing ratios. Our results also indicate that dialysis centers that offer PD are not more likely to realize better health outcomes, measured in terms of SHR. In summary, Column 3 exhibits the overall effects of dialysis center characteristics and practice patterns on SHR.

\subsection{Mediation effect}

Based on a comparison of the results reported in Table V, we find evidence of full mediation. Specifically, we find that the magnitudes of the coefficients of some predictor variables are weaker when we compare the estimates in Columns 1 and 3. For example, the coefficients of Treatment/patient and Cost/patient are statistically significant in Column 1 but are not significant in the full model results shown in Column 3. The reduction in the strength of their association with SHR indicates that their effect on SHR is completely mediated through the intermediate dialysis process measures. The full mediation effect between treatment per patient and SHR suggests that scale economics benefit patient outcomes primarily through improvements in dialysis process measures. Specifically, more dialysis treatments enable clinicians and dialysis centers to make more efficient use of clinical resources which are, in turn, associated with improvements in the dialysis process measures. Similarly, the relationship between treatment cost per patient and SHR is completely mediated by clinical process measures, possibly because dialysis centers that have high composite cost admit more severe patients. These patients have worse clinical outcomes which further impact SHR. Similarly, we observe that among practice patterns, the coefficient of station-to-patient ratio is statistically significant in the first model but is insignificant in the full model. Again, this result suggests that the effect of station-to-patient ratio on SHR is fully mediated through the process measures. A possible reason for this mediation effect may be that the main benefit of having more available dialysis stations is observed through improvements in dialysis adequacy and anemia management measures.

Our results indicate that the effects of facility and practice pattern variables are partially mediated through the dialysis process measures. For instance, the coefficients of Catheter Usage in Columns 1 and 3 are statistically significant, but the magnitude of the coefficients in Column 3 is reduced from 0.0051 to 0.0046 after including the clinical process variables. This suggests that the impact of Catheter Usage is partially mediated by clinical processes. 
The same pattern exists in Late Shift, as we observe that its coefficients weaken from 0.073 to 0.070 . The partial mediation effect reveals that the downside of offering late night shift in dialysis centers also affects patient outcome directly through reductions in their process measures. Furthermore, Own Type is also partially mediated through clinical processes, suggesting that for-profit dialysis centers are not only likely to exhibit greater hospitalization ratios, but also poorer performance related to their dialysis process measures.

Based on these results, we explore the key relationships of interest among practice patterns, clinical processes and SHR and show the existence of either full or partial mediation

Antecedents of patient health outcomes

43 effects as delineated in Figure 1. Our research constitutes an exploratory study of the antecedents of health outcomes among patients who are treated at dialysis centers across the USA.

\section{Discussion}

Beginning on January 1, 2011, Medicare launched the bundled payment system with pay-for-performance initiatives. The intent was to control unnecessary costs in dialysis services and improve the quality of care. The two metrics that are part of the dialysis QIP are urea clearance and Hgb levels. One of our objectives is to study how dialysis facility and practice characteristics impact clinical process quality measures and patient outcomes - a topic that has not been studied using recent data from a large sample of dialysis clinics. We further investigate the interdependent relationships among practice characteristics, clinical process measures and patient outcomes, thereby bringing new insights about the mechanisms through which facility characteristics and practice patterns impact patient outcomes.

Under the bundled PPS, providers receive a fixed payment for each treatment per patient, including the actual cost of dialysis and all injectable medications or their oral equivalents. To ensure cost-efficient treatment, dialysis centers have to optimally assign their limited budget and resources to ensure that the actual expenditure per treatment does not rise above the bundled payment threshold, while maintaining the quality of care levels with respect to clinical process measures. In the new payment reform environment, the costs of adopting a practice pattern need to be weighed against the potential benefits of adoption with respect to the quality of care measures. Our research provides several implications for facilities to lower their risk of being penalized and reduce treatment expenditures, as well as insights for health policy makers.

First, our results show that although dialyzer reuse is positively associated with clinical process measures, they are also likely to be associated with greater hospitalization risk. We note that four of the top ten dialysis providers in the USA engage in dialyzer reuse practice[15]. The primary benefit of dialyzer reuse is economic in terms of money and time. The cost savings incurred from dialyzer reuse are significant and can be used to improve the URR and Kt/V ratios. However, dialyzer reuse may be associated with an increase in the number of infections and require preventive interventions. Previous researchers (Lacson and Lazarus, 2006; Twardowski, 2006) have suggested that dialyzers can be used safely but only under the condition that dialysis practices follow strict standards. Hence, the economic benefits of dialyzer reuse need to be carefully weighed against their clinical risk.

Second, our results suggest that facilities should optimize their staffing levels with respect to physicians and nurses, so that they do not compromise dialysis quality measures, while continuing to maintain treatment costs under the acceptable bundled payment limits. Having more nurses on site is helpful to patients, but under the condition that dialysis facilities provide nurses with sufficient support to provide adequate 
dialysis services. For small free-standing dialysis centers, having more physicians may not be cost-effective in improving clinical outcomes because they are serving few patients but consume excessive resource. Given the limited amount of resources available to free-standing dialysis facilities, management need to balance staffing and other resources in an efficient manner.

Third, offering late shifts may not achieve the expected result of improving patient outcomes, but may indeed be associated with lower levels of dialysis adequacy and higher hospitalization risk, because such arrangements may negatively impact staff satisfaction and increase the risk of medical errors. Therefore, dialysis centers may need to rethink their strategy of offering late shift sessions. Furthermore, our results suggest that dialysis facilities should consider increasing their market share, through mergers or acquisitions, as dialysis clinics with greater local market share are likely to exhibit lower SHR.

\section{Conclusions}

In this paper, we examine the interdependent associations among practice patterns, clinical processes and patient outcomes in the dialysis industry. We find that dialyzer reuse is positively associated with clinical process measures but is also associated with higher hospitalization risk. A higher nurses-to-patient ratio improves anemia management, whereas higher physician-to-patient ratio increases the likelihood of poor anemia control. While the nurse and physician caseloads are not significantly associated with patient hospitalization risk, we observe that the station-to-patient ratio improves dialysis adequacy and hospitalization risk. Offering PD as an optional modality is not preferable. It deteriorates clinical processes but does not significantly raise hospitalization risk. Providing late shifts reduces the percentage of patients who receive adequate dialysis and increases their hospitalization risk. Furthermore, our results show that facility ownership type, prolonged catheter usage and late shift have both direct and indirect associations with patient outcomes (measured as SHR) and are partially mediated through dialysis processes. We also find that the impact of treatment cost per patient, treatment volume per patient and station ratio becomes insignificant, after controlling for clinical processes, thereby suggesting the existence of full mediation.

In spite of the large data set used to conduct this research, our study also has several limitations. First, the observed relationships should be interpreted as associations instead of causal relationships, even though we control for several facility characteristics and used lagged variables to mitigate potential simultaneity bias. Second, although the SHR provided by CMS has been adjusted for case mix factors, we cannot adjust for other possible factors such as severity of patient illness. Patients may switch to other dialysis facilities with better performance if their current providers do not offer high-quality care. Hence, some dialysis facilities may exhibit a larger mix of patients with severe conditions, which could result in poorer outcomes. We note that this concern may be partially mitigated, as patients are attributed to a facility only after receiving treatment for at least 60 days.

Third, as our results are based on the general population of US dialysis centers, and the study was conducted using dialysis facility as the unit of analysis, they may not be generalizable to patient-level treatment recommendations. Fourth, as our analyses were based on observational facility-level data, we are unable to account for patient-specific factors such as patient behavior after discharge, socioeconomic status, insurance type and nutrition status, as well as potential facility confounding variables like technology adoption. Finally, our data are restricted to free-standing dialysis facilities with HD, and 
hence, may not be generalizable to hospital-based dialysis centers and PD patients. Future research could be expanded in scope to address these limitations to derive greater insights for patient treatment.

Our research represents an exploratory study to examine the general associations between dialysis center practice patterns and facility clinical process measures. Based on our findings, future field studies can be designed to explore the mechanisms through which practice patterns can affect clinical process decisions and establish causality among the key variables of interest, to expand the generalizability of our results.

\section{Notes}

1. Available at: www.usrds.org/2014/view/v2_01.aspx

2. Available at: https://innovation.cms.gov/initiatives/comprehensive-esrd-care/

3. Available at: www.cms.gov/Research-Statistics-Data-and-Systems/Downloadable-Public-UseFiles/Cost-Reports/RNL-2011-form.html

4. The top five dialysis providers in the USA operate a chain of dialysis clinics for more than 400,000 patients annually. www.nephrologynews.com/largest-dialysis-providers-2016-poised-change/

5. Available at: www.qualitymeasures.ahrq.gov/tutorial/HealthOutcomeMeasure.aspx

6. Medicare publishes claims data and SHR data from different periods. For example, in the data set published on May 6, 2015, claims data are from 07/01/2013-06/30/2014, while SHR data are from 01/01/2013-12/31/2013.

7. We also test our results using the first four digits of zip code. The results are qualitatively consistent.

8. Available at: http://dialysispatients.org/sites/default/files/menus/FINAL $\% 2009 \% 2012 \% 2012 \%$ 20Patient $\% 20$ Satisfaction $\% 20$ Survey $\% 20$ Results.pdf

9. We use Nurse-To-Patient Ratio in all equations. Similar pattern applies to Physician-to-patient ratio and Station-to-patient ratio.

10. Available at: www.cms.gov/Medicare/Quality-Initiatives-Patient-Assessment-Instruments/ESRD QIP/Downloads/ESRD-QIP-Sumary-PY2012-16.pdf

11. For example, educational documents from NIDDK available at www.niddk.nih.gov/healthinformation/health-topics/kidney-disease/hemodialysis-dose-and-adequacy/Pages/facts.aspx

12. As shown in Table II, the nurse-to-patient ratio is small in magnitude. The average nurse-to-patient ratio is 0.0726 , which is equivalent to 13.77 patients per nurse. We treat 14 patients per nurse as a benchmark. Hence, reducing one patient per nurse will increase the nurse-to-patient ratio by 0.0055 , which we consider as one unit of increase in nurse-to-patient ratio. We also use interpret the coefficients of physician-to-patient ratio and station-to-patient ratio in a similar manner.

13. For physician-to-patient ratio, the benchmark is 256 patients to each physician reduction of 10 patients per physician increases physician-to-patient ratio by 0.000159 . We consider this amount as equivalent to one unit of measure for physician-to-patient ratio.

14. For station-to-patient ratio, the benchmark is 3 patients per station. With a reduction of one patient per station, the station-to-patient ratio increases by 0.1667 , which we consider as one unit of measure.

15. Available at: www.nephrologynews.com/largest-dialysis-providers-2016-poised-change/

\section{References}

Aiken, L.H., Clarke, S.P. and Sloane, D.M. (2002), "Hospital staffing, organization, and quality of care: cross-national findings", International Journal for Quality in Health Care, Vol. 14 No. 1, pp. 5-14.

Allison, P.D. (2001), Missing Data (Vol. 136), Sage Publications, New York, NY.
Antecedents of patient health outcomes 
Baron, R.M. and Kenny, D.A. (1986), "The moderator-mediator variable distinction in social psychological research: Conceptual, strategic, and statistical considerations", Journal of Personality and Social Psychology, Vol. 51 No. 6, pp. 11-73.

Bell, C.M., Urbach, D.R., Ray, J.G., Bayoumi, A., Rosen, A.B., Greenberg, D. and Neumann, P.J. (2006), "Bias in published cost effectiveness studies: systematic review", Bmj, Vol. 332 No. 7543, pp. 699-703.

Centers for Medicare and Medicaid Services (2015), Comprehensive ESRD Care Model, available at: https://innovation.cms.gov/initiatives/comprehensive-esrd-care/ (accessed 4 October 2017).

Chandrashekar, A., Ramakrishnan, S. and Rangarajan, D. (2014), "Survival analysis of patients on maintenance hemodialysis", Indian Journal of Nephrology, Vol. 24 No. 4, p. 206.

Dalal, P., Sangha, H. and Chaudhary, K. (2011), "In peritoneal dialysis, is there sufficient evidence to make "PD First” Therapy?”, International Journal of Nephrology, Vol. 1.

Feldman, H.I., Bilker, W.B., Hackett, M., Simmons, C.W., Holmes, J.H., Pauly, M.V. and Escarce, J.E.J. (1999), "Association of dialyzer reuse and hospitalization rates among hemodialysis patients in the US”, American Journal of Nephrology, Vol. 19 No. 6, pp. 641-648.

Garred, L.J., Canaud, B., Flavier, J.L., Poux, C., Polito-Bouloux, C. and Mion, C. (1990), "Effect of reuse on dialyzer efficacy", Artificial Organs, Vol. 14 No. 2, pp. 80-84.

Greene, W.H. (2003), Econometric Analysis, Prentice Hall, New York, NY.

Harley, K.T., Streja, E., Rhee, C.M., Molnar, M.Z., Kovesdy, C.P., Amin, A.N. and Kalantar-Zadeh, K. (2013), "Nephrologist caseload and hemodialysis patient survival in an urban cohort", Journal of the American Society of Nephrology, Vol. 24 No. 10, pp. 1678-1687.

Held, P.J., Wolfe, R.A., Gaylin, D.S., Port, F.K., Levin, N.W. and Turenne, M.N. (1994), "Analysis of the association of dialyzer reuse practices and patient outcomes", American Journal of Kidney Diseases, Vol. 23 No. 5, pp. 692-708.

Held, P.J., Port, F.K., Wolfe, R.A., Stannard, D.C., Carroll, C.E., Daugirdas, J.T., Bloembergen, W.E., Greer, J.W. and Hakim, R.M. (1996), "The dose of hemodialysis and patient mortality", Kidney International, Vol. 50 No. 2, pp. 550-556.

Hirth, R.A., Turenne, M.N., Wheeler, J.R., Nahra, T.A., Sleeman, K.K., Zhang, W. and Messana, J.A. (2013), "The initial impact of Medicare's new prospective payment system for kidney dialysis", American Journal of Kidney Diseases, Vol. 62 No. 4, pp. 662-669.

Hollenbeck, M., Niehuus, A., Wozniak, G. and Hennigs, S. (2012), "CCentral venous catheters as access for acute and long-term dialysis]", Der Chirurg; Zeitschrift fur alle Gebiete der operativen Medizen, Vol. 83 No. 9, pp. 801-808.

Lacson, E. and Lazarus, J.M. (2006), "Unresolved issues in dialysis: dialyzer best practice: single use or reuse?", Seminars in Dialysis, Vol. 19 No. 2, pp. 120-128.

Leypoldt, J.K., Cheung, A.K. and Deeter, R.B. (1998), "Effect of hemodialyzer reuse: dissociation between clearances of small and large solutes", American journal of kidney diseases, Vol. 32 No. 2, pp. 295-301.

Lowrie, E.G., Li, Z., Ofsthun, N. and Lazarus, J.M. (2004), "Reprocessing dialysers for multiple uses: recent analysis of death risks for patients", Nephrology Dialysis Transplantation, Vol. 19 No. 11, pp. 2823-2830.

McClellan, W.M., Soucie, J.M. and Flanders, W.D. (1998), "Mortality in end-stage renal disease is associated with facility-to-facility differences in adequacy of hemodialysis", Journal of the American Society of Nephrology, Vol. 9 No. 10, pp. 1940-1947.

O'hare, A.M., Johansen, K.L. and Rodriguez, R.A. (2006), "Dialysis and kidney transplantation among patients living in rural areas of the United States", Kidney International, Vol. 69 No. 2, pp. 343-349. 
Ouseph, R., Smith, B.P. and Ward, R.A. (1997), "Maintaining blood compartment volume in dialyzers reprocessed with peracetic acid maintains Kt/V but not $\beta 2$-microglobulin removal", American Journal of Kidney Diseases, Vol. 30 No. 4, pp. 501-506.

Owen, W.F. Jr, Lew, N.L., Liu, Y., Lowrie, E.G. and Lazarus, J.M. (1993), “The urea reduction ratio and serum albumin concentration as predictors of mortality in patients undergoing hemodialysis", New England Journal of Medicine, Vol. 329 No. 14, pp. 1001-1006.

Parker, T.F., Husni, L., Huang, W., Lew, N., Lowrie, E.G. and Associates, D.N. (1994), "Survival of hemodialysis patients in the United States is improved with a greater quantity of dialysis", patient health outcomes American Journal of Kidney Diseases, Vol. 23 No. 5, pp. 670-680.

Pisoni, R.L., Bragg-Gresham, J.L., Young, E.W., Akizawa, T., Asano, Y., Locatelli, F., Bommer, J., Cruz, J.M., Kerr, P.G., Mendelssohn, D.C., Held, P.J. and Port, F.K. (2004), “Anemia management and outcomes from 12 countries in the dialysis outcomes and practice patterns study (DOPPS)", American Journal of Kidney Diseases, Vol. 44 No. 1, pp. 94-111.

Port, F.K., Wolfe, R.A., Hulbert-Shearon, T.E., Daugirdas, J.T., Agodoa, L.Y., Jones, C., Orzol, S.M. and Held, P.J. (2001), "Mortality risk by hemodialyzer reuse practice and dialyzer membrane characteristics: results from the USRDS dialysis morbidity and mortality study", American Journal of Kidney Diseases, Vol. 37 No. 2, pp. 276-286.

Pozniak, A.S., Hirth, R.A., Banaszak-Holl, J. and Wheeler, J.R. (2010), "Predictors of chain acquisition among independent dialysis facilities", Health Services Research, Vol. 45 No. 2 , pp. 476-496.

Pronovost, P.J., Dang, D., Dorman, T., Lipsett, P.A., Garrett, E., Jenckes, M. and Bass, E.B. (2001), "Intensive care unit nurse staffing and the risk for complications after abdominal aortic surgery", Effective Clinical Practice, Vol. 4 No. 5, pp. 199-206.

Sens, F., Schott-Pethelaz, A.M., Labeeuw, M., Colin, C. and Villar, E. (2011), "Survival advantage of hemodialysis relative to peritoneal dialysis in patients with end-stage renal disease and congestive heart failure", Kidney International, Vol. 80 No. 9, pp. 970-977.

Szczech, L.A., Klassen, P.S., Chua, B., Hedayati, S.S., Flanigan, M., McClellan, W.M., Reddan, D.N., Rettig, R.A., Frankenfield, D.L. and Owen, W.F. (2006), "Associations between CMS's clinical performance measures project benchmarks, profit structure, and mortality in dialysis units", Kidney International, Vol. 69 No. 11, pp. 2094-2100.

Thomas-Hawkins, C., Flynn, L. and Clarke, S.P. (2008), "Relationships between registered nurse staffing, processes of nursing care, and nurse-reported patient outcomes in chronic hemodialysis units”, Nephrology Nursing Journal: Journal of the American Nephrology Nurses' Association, Vol. 35 No. 2, p. 123.

Twardowski, Z.J. (2006), "Dialyzer reuse - part II: advantages and disadvantages”, Seminars in Dialysis, Vol. 19 No. 3, pp. 217-226.

Upadhyay, A., Sosa, M.A. and Jaber, B.L. (2007), "Single-use versus reusable dialyzers: the known unknowns", Clinical Journal of the American Society of Nephrology, Vol. 2 No. 5, pp. 1079-1086.

Wingard, R.L., Pupim, L.B., Krishnan, M., Shintani, A., Ikizler, T.A. and Hakim, R.M. (2007), "Early intervention improves mortality and hospitalization rates in incident hemodialysis patients: RightStart program", Clinical Journal of the American Society of Nephrology, Vol. 2 No. 6, pp. $1170-1175$.

Wooldridge, J. (2012), Introductory Econometrics: A Modern Approach, Cengage Learning, Boston.

Yan, G., Norris, K.C., Xin, W., Ma, J.Z., Alison, J.Y., Greene, T., Wei, Y. and Cheung, A.K. (2013), “Facility size, race and ethnicity, and mortality for in-center hemodialysis", Journal of the American Society of Nephrology, Vol. 24 No. 12, pp. 2062-2070. 
Yang, J.Y., Chen, L., Chao, C.T., Peng, Y.S., Chiang, C.K., Kao, T.W., Chien, K.L., Wu, H.Y., Huang, J.W. and Hung, K.Y. (2015), "Comparative study of outcomes among patients with polycystic kidney disease on hemodialysis and peritoneal dialysis", Scientific Reports, Vol. 5.

Zhang, Y., Cotter, D.J. and Thamer, M. (2011), "The effect of dialysis chains on mortality among patients receiving hemodialysis", Health Services Research, Vol. 46 No. 3, pp. 747-767.

\section{Corresponding author}

Indranil Bardhan can be contacted at: bardhan@utdallas.edu

For instructions on how to order reprints of this article, please visit our website:

www.emeraldgrouppublishing.com/licensing/reprints.htm

Or contact us for further details: permissions@emeraldinsight.com 